\title{
CENTROS ESPECIALES DE EMPLEO DE EUSKADI. EL MODELO VASCO DE INCLUSIÓN SOCIOLABORAL
}

\author{
Ane Echebarria Rubio \\ Investigadora del Instituto GEZKI \\ Universidad del País Vasco
}

\section{RESUMEN}

El empleo se ha convertido en uno de los principales instrumentos de inclusión social de las personas con discapacidad. La inclusión laboral supone el derecho a trabajar en igualdad de condiciones con las demás personas, en un entorno abierto, accesible e inclusivo, y mediante un trabajo libremente elegido. Los Centros Especiales de Empleo de Euskadi, que conforman el llamado Modelo Vasco de Inclusión Sociolaboral, llevan años trabajando con el objetivo de crear empleo de calidad para las personas con más necesidades de apoyo y facilitar el tránsito al mercado de empleo ordinario para el colectivo. En el presente estudio, se han analizado las cualidades de los centros especiales de empleo para el desarrollo profesional y personal de las personas con discapacidad, poniendo una especial atención en el funcionamiento y características de las entidades pertenecientes al llamado Modelo Vasco de Inclusión Sociolaboral.

PALABRAS CLAVE: Centros especiales de empleo, discapacidad, inclusión, empleo, Modelo Vasco de Inclusión Sociolaboral.

CLAVES ECONLIT: A13, J710, K310.

Cómo citar este artículo/How to cite this article: ECHEBARRIA RUBIO, A.: “Centros especiales de empleo de Euskadi. El modelo vasco de inclusión sociolaboral", CIRIEC-España, Revista Jurídica de Economía Social y Cooperativa, nº 36, 2020, pp. 153-175. D0I: 10.7203/ CIRIEC-JUR.36.17249. 


\section{SHELTERED EMPLOYMENT ORGANIZATIONS FROM EUSKADI. THE BASQUE MODEL OF SOCIAL AND LABOUR INCLUSION}

\section{EXPANDED ABSTRACT}

Employment can be considered as one of the most efficient tools for the social inclusion for people with disabilities. The labour inclusion involves, the right of people with disabilities to work on an equal basis with others, through freely chosen work and in one inclusive, open and accessible environment. Sheltered Employment Organizations (SEO) from the Basque Country, that constitute the Basque Model of Social and Labour Inclusion, have years of experience in this sector, and they have a common fundamental aim, which is to generate quality employment for people with more need for support and to promote insertion into the open labour market. The aim of this study is to analyse the employment of people with disabilities, to then focus on the characteristics of the Basque Model of Social and Labour Inclusion, highlighting the positive elements as well as the future challenges.

The concept of disability is defined by the General Law on the Rights of People with Disabilities and their Inclusion (Ley General de Derechos de las Personas con Discapacidad- LGDPCD in its Spanish initials). Article 2.a) establishes that disability is the interaction between a person with a deficiency and the barriers that prevent their full and effective participation in society. This concept is based on the definition of person with disability defined at the UN Convention on the Rights of Persons with Disabilities (CRPD). Therefore, focus is placed on social barriers, and the society's impossibility to develop means of support to bypass them.

Article 4 of the LGDPCD says that "People with disabilities are those who show physical, mental, intellectual or sensory deficiencies, which are presumably permanent and which, when interacting with different barriers, may prevent their full and effective participation in society on an equal basis with others".

Full and effective inclusion into society of people with disabilities involves, among other things, the right of these people to work on an equal basis with others. Regarding national regulations, public authorities must carry out a prevision, treatment, rehabilitation and integration policy for people with disabilities, providing them with the specific care that they require and protecting them so that they may enjoy the rights afforded to all citizens in the Constitution.

Two types of employment can be differentiated through which the collective can exercise their right to work: regular employment, which includes working for others (both in the pri- 
vate and public sectors), self-employment and collective self-employment; and employment in Sheltered Employment Organizations. Lastly, the LGDPCD offer occupational therapy services in order to achieve maximum personal development and, when possible, facilitate training and preparation for access to employment.

Sheltered Employment Organizations are the main facilitating instrument for access to employment for people with disabilities. They are companies that carry out productive activity in goods and services, and who regularly compete in the market. For this reason, they will be subjected to the same rules and requirements as other companies in the sector to which they belong. Article 43 of the LGDPCD establishes the two mandatory requirements that these organizations must fulfil: 1 ) they must have a minimum of $70 \%$ of the staff comprising working people with a recognized degree of disability equal to or greater than $33 \%$ and, 2) they must provide personal and social adjustment services required by the working people through support units created for such a purpose.

Sheltered Employment Organizations from the Basque Country, constitute the Basque Model of Social and Labour Inclusion (all of them are social initiative SEO and it concentrates 95\% of all employment initiatives in the Autonomous Community of the Basque Country). With years of experience on this sector, they have a common fundamental aim, which is to generate quality employment for people with disabilities, prioritizing those with more need for support, and to promote insertion into the open labour market. In addition, all of them share each of the guiding principles of the Social Economy.

The Basque Model is a model focused on people's abilities and their development, generating employment opportunities in professional and competitive environments. The entire professional itinerary is carried out with an inclusive approach. They manage different employment programmes and have full labour insertion itineraries, that are adapted to the needs and wishes of each person. It is considered a flexible and innovative model, and they work each day for excellence in the management of different types of business activities, demonstrating the ability of people with disabilities to carry out different jobs. This social initiative SEO works towards inclusive, open and accessible employment in equal conditions. The ultimate goal is that people with disabilities are able to earn a living through a freely chosen job.

At this point, it is very important to analyse the Basque Model of Social and Labour Inclusion in light of the International Convention on the Rights of People with Disabilities, that was conceived as a human rights instrument with the explicit dimension of social development and an inclusive society. It reaffirmed the fact that all people, regardless of their disability, must be able to enjoy all human rights and fundamental freedoms. Article 27 of the Convention establishes that people with disabilities have the right to work and to earn a living on 
an equal basis with others, through a freely chosen job. The analyses of this article shows that the Basque Model is a clear example of the working environment defended by the International Convention. They have managed to build an accessible, open and inclusive environment for people with disabilities, where they can freely carry out the job they have chosen.

In the Basque Country, employment has been a fundamental tool in the inclusion of people with disabilities. The daily work and effort of the organizations of the Basque Model of Social and Labour Inclusion contribute directly to the inclusion of the group, and they work to create a decent and quality employment. Additionally, there are several challenges facing the future of the SE0, since there is a long way to go to achieving more and better employment for people with disabilities, especially for people with the greatest need for support.

KEYWORDS: Sheltered employment organizations, disability, inclusion, employment, Basque Model of Social and Labour Inclusion. 


\section{SUMARIO}

1. Introducción. 2. El empleo de las personas con discapacidad. 2.1. Conceptos: discapacidad y persona con discapacidad. 2.2. Normativa. 3. Tipos de empleo. 3.1. Empleo ordinario. 3.2. Empleo en Centros Especiales de Empleo. 3.3. Servicio ocupacional. 4. Los Centros Especiales de Empleo de iniciativa social en la Comunidad Autónoma de Euskadi. El Modelo Vasco de Inclusión Sociolaboral. 4.1. El modelo vasco a la luz de la Convención de la ONU. 5. Conclusiones. Bibliografía.

\section{Introducción}

El empleo es una de las herramientas más eficaces para la inclusión social de las personas con discapacidad. Se trata de un derecho universal, que brinda a las personas autonomía e integración en la sociedad, además de ayudar a visibilizar al colectivo.

La aprobación de la Convención Internacional sobre los Derechos de las Personas con Discapacidad (en adelante, CDPD) de las Naciones Unidas ${ }^{1}$, marcó una nueva era en el modelo de tratamiento de los derechos del colectivo. El texto nació con el objetivo de luchar contra la discriminación y conseguir el reconocimiento de los derechos de las personas con discapacidad. Esto supuso una apuesta por una sociedad inclusiva, que aborda la discapacidad desde el modelo social y desde un enfoque de derechos humanos. Tal y como establece dicha Convención, la plena inclusión en la sociedad implica, entre otras cosas, el derecho de las personas con discapacidad a trabajar en igualdad de condiciones con las demás, en un entorno abierto, accesible e inclusivo, y mediante un trabajo libremente elegido. Se trata de una pieza clave en el camino de la inclusión social, y es por esta razón, por la que ha sido uno de los objetivos fundamentales de las políticas activas.

Los Centros Especiales de Empleo (en adelante, CEE), han sido y son el principal instrumento facilitador del acceso al empleo del colectivo en la CAE, cuya finalidad es asegurar un trabajo productivo y remunerado a las personas con discapacidad, a la vez que son un medio de inclusión en el empleo ordinario.

En la Comunidad Autónoma de Euskadi (CAE), debe hablarse del Modelo Vasco de Inclusión Sociolaboral, gestionado por CEE de iniciativa social y pública, y que se 
ha convertido en un referente a nivel europeo. Concentra el 95\% de todas las iniciativas de empleo del territorio y cuenta con más de 10.000 personas con discapacidad empleadas. Es un modelo centrado en las capacidades de cada una de las personas y en su desarrollo personal y profesional.

Este trabajo pretende analizar las características y cualidades de los Centros Especiales de Empleo en la inclusión laboral de las personas con discapacidad. Se centrará en el análisis del Modelo Vasco de Inclusión Sociolaboral, con el fin de conocer las peculiaridades que lo han llevado a convertirse en un referente en este ámbito.

El estudio partirá de una reflexión sobre las personas con discapacidad y la normativa vigente entorno al sector. A continuación, se analizarán los diferentes tipos de empleo, aportando datos sobre la realidad de cada uno de ellos y poniendo especial atención en el empleo protegido llevado a cabo en los Centros Especiales de Empleo y sus características. La tercera parte del trabajo se centrará en las peculiaridades del Modelo Vasco de Inclusión Sociolaboral, apartado en el que se realizará una comparativa con el modelo social que reclama la Convención de la ONU. El texto concluirá con una reflexión sobre los elementos positivos y los retos a los que se enfrentan los CEE, y en particular, las entidades de nuestro territorio, para lograr la igualdad y el respeto de los derechos de las personas con discapacidad.

En cuanto a la metodología utilizada, el estudio se ha llevado a cabo, por un lado, mediante el análisis de la legislación y normativa vigente y la revisión bibliográfica de diferentes expertos en la materia, y por otro, por medio del análisis de datos estadísticos provenientes de diversas fuentes oficiales y no oficiales. Cabe destacar, que el presente trabajo parte de un Informe reciente sobre el Modelo Vasco de Inclusión Sociolaboral ${ }^{2}$.

\section{El empleo de las personas con discapacidad}

\subsection{Conceptos: discapacidad y persona con discapacidad}

Antes de analizar el empleo de las personas con discapacidad, conviene delimitar y aclarar, como cuestiones previas, los conceptos de discapacidad y persona con discapacidad.

En los últimos ańos, el modelo de tratamiento de la discapacidad ha experimentado una notable evolución, ya que se ha ido dejando atrás el enfoque asistencial y paternalista que ponía el foco en las deficiencias de las personas, y se ha centrado en el modelo social, basado en los derechos humanos y cuyo objetivo es "la normaliza- 
ción de la sociedad, para hacer frente a las necesidades de todas las personas" (García, 2011, 31).

En la actualidad, es la Ley General de derechos de las personas con discapacidad y de su inclusión social (en adelante, LGDPCD) ${ }^{3}$ la encargada de definir la discapacidad. La norma, que acoge el modelo social y deja atrás el referente médico ${ }^{4}$ de las anteriores $^{5}$, tal y como reivindicaba el movimiento asociativo de la discapacidad (De Fuentes, 2016, 83), establece en el artículo 2.a) que la discapacidad "es una situación que resulta de la interacción entre las personas con deficiencias previsiblemente permanentes y cualquier tipo de barreras que limiten o impidan su participación plena y efectiva en la sociedad, en igualdad de condiciones con las demás". De esta manera, se pone el foco en las barreras de la sociedad y en la imposibilidad de articular medidas de apoyo suficientes para superarlas.

En cuanto al concepto de persona con discapacidad, es un término que también ha sido objeto de una notable transformación a lo largo de estos años ${ }^{6}$ y que se encuentra totalmente consolidado en el ámbito del Derecho (Bengoetxea, 2014, 529), a partir de la entrada en vigor de la Ley de dependencia del $2006^{7}$. Así, el artículo 4 de la LGDPCD dispone que tendrá la consideración de persona con discapacidad "quien presente deficiencias fisicas, mentales, intelectuales o sensoriales, previsiblemente permanentes que, al interactuar con diversas barreras, puedan impedir su participación plena y efectiva en la sociedad, en igualdad de condiciones con los demás" (con independencia de tener un reconocimiento previo administrativo). Y añade que, además, "tendrán la consideración de personas con discapacidad aquellas a quienes se les haya reconocido un grado de discapacidad igual o superior al 33 por ciento. Se considerará que presentan una discapacidad en grado igual o superior al 33 por ciento los pensionistas de la Seguridad Social que tengan reconocida una pensión de incapacidad permanente en el grado de total, absoluta o gran invalidez, y a los pensionistas de clases pasivas que tengan

3. Real Decreto Legislativo 1/2013, de 29 de noviembre, por el que se aprueba el Texto Refundido de la Ley General de derechos de las personas con discapacidad y de su inclusión social.

4. Se alude a la discapacidad en términos de enfermedad, y el tratamiento social se basa en una actitud paternalista, enfocada hacia las deficiencias de las personas (Toboso, M. y Arnau, M.S., 2008:67).

5. Ley 13/1982, de 7 de abril de integración social de las personas con discapacidad-LISMI.

6. Ha superado diversos términos como minusválido, inválido o disminuido, presentes en anteriores normas.

7. La Ley 39/2006, de 14 de diciembre, de Promoción de la Autonomía Personal y Atención a las personas en situación de dependencia, establece que "a partir de la entrada en vigor de la presente Ley, las disposiciones normativas elaboradas por las Administraciones Públicas utilizarán los términos "persona con discapacidad» o "personas con discapacidad" para denominarlas" (Disposición Adicional Octava). 
reconocida una pensión de jubilación o de retiro por incapacidad permanente para el servicio o inutilidad".

En este punto, cabe destacar el colectivo de personas con discapacidad con mayores necesidades de apoyo, que según el artículo 3.1 del Real Decreto 469/2006, son aquellas con discapacidad física o sensorial en un grado mínimo del 65\%; y las que tengan parálisis cerebral, enfermedad mental o intelectual con un grado igual o superior al $33 \%$.

\subsection{Normativa}

La Asamblea de las Naciones Unidas aprobó el 13 de diciembre de 2006 la Convención Internacional sobre los Derechos de las Personas con Discapacidad, el cual marcó un antes y un después en el tratamiento de la discapacidad. Supuso un paso significativo hacia una sociedad inclusiva, que lucha contra la discriminación y por la plena inclusión del colectivo. El modelo de tratamiento que plantea esta Convención, establece que la inclusión implica, entre otras cosas, el derecho de las personas con discapacidad a trabajar en igualdad de condiciones con las demás, mediante un trabajo libremente elegido (artículo 27). Este mismo artículo, insta a los Estados miembro a adoptar medidas para prohibir la discriminación y asegurar la igualdad de oportunidades, un hecho que llevó a los legisladores estatales a adaptar la normativa en materia de empleo de las personas con discapacidad.

En la misma línea que las Naciones Unidas se ha pronunciado también la Unión Europea, a través de la Directiva 2000/78/CE de 27 de noviembre, que tiene el objetivo de luchar contra la discriminación por motivos de discapacidad, entre otros, en el ámbito del empleo.

En cuanto a la normativa del estado español, debe destacarse como base de todas las normas que se aprueben en esta materia el artículo 9.2 de la Constitución, que establece que los poderes públicos tendrán la obligación de promover las condiciones para que la libertad y la igualdad del individuo sean reales y efectivas, además de remover los obstáculos que impidan o dificulten su plenitud y facilitar la participación de todos los ciudadanos en la vida económica y social.

Por otro lado, la norma clave que regula el derecho al trabajo de las personas con discapacidad es la Ley General de derechos de las personas con discapacidad y de su inclusión social. En materia de empleo, cabe subrayar el artículo 7.3, en el que requiere a las Administraciones públicas una protección especialmente intensa en esta materia. Además, el artículo 35 dispone que "las personas con discapacidad tienen derecho al trabajo, en condiciones que garanticen la aplicación de los principios de igualdad 
de trato y no discriminación", y el artículo 37 obliga a las Administraciones públicas a fomentar las oportunidades de empleo y promover los apoyos necesarios para la obtención y mantenimiento de éste, mediante políticas que tengan como finalidad aumentar la tasa de actividad y de ocupación e inserción laboral, así como mejorar la calidad del empleo, combatiendo activamente su discriminación. Por último, el artículo 39 enumera algunas técnicas de acción positiva que pueden ayudar a la inclusión laboral de las personas con discapacidad, como la adaptación de los puestos de trabajo, bonificaciones en las cuotas de la Seguridad Social y cuantas otras se consideran adecuadas para promover la colocación de las personas con discapacidad, especialmente la promoción de cooperativas y otras entidades de la economía social.

\section{Tipos de empleo}

Las personas con discapacidad pueden ejercer su derecho al trabajo a través de dos tipos de empleo. El primero de ellos es el empleo ordinario (EO), dentro del cual pueden diferenciarse el trabajo por cuenta ajena (tanto en el sector público como en el privado), el empleo autónomo y el autoempleo colectivo o trabajo asociado. El segundo es el empleo protegido, en el que entran los Enclaves Laborales ${ }^{8}$ y el empleo en Centros Especiales de Empleo, en el cual se centrará el presente trabajo.

Cabe destacar la opción del servicio ocupacional (SO), llevado a cabo en centros ocupacionales y los cuales ofrecen servicios de ajuste personal y social con el fin de lograr el máximo desarrollo personal del colectivo, y en los casos en los que fuera posible, facilitar su capacitación y preparación para el acceso al empleo (artículo 52 LGDPCD). Se trata de un servicio social, por lo que no existe relación laboral.

A continuación, se analizarán las principales características de cada uno de estos tipos de empleo, además se aportarán datos que ofrezcan una fotografía de la realidad del sector en Euskadi y se pondrá una especial atención en el empleo en Centros Especiales de Empleo.

8. Regulados en el Real Decreto 290/2004, de 20 de febrero, por el que se regulan los enclaves laborales como medida de fomento del empleo de las personas con discapacidad. Artículo 1.2 "Se entiende por enclave laboral el contrato entre una empresa del mercado ordinario de trabajo, llamada empresa colaboradora, y un centro especial de empleo para la realización de obras o servicios que guarden relación directa con la actividad normal de aquélla y para cuya realización un grupo de trabajadores con discapacidad del centro especial de empleo se desplaza temporalmente al centro de trabajo de la empresa colaboradora". 


\subsection{Empleo ordinario}

Dentro del empleo ordinario se pueden identificar los siguientes: el llevado a cabo por cuenta ajena en empresas privadas o en las Administraciones públicas, el empleo autónomo y el autoempleo colectivo (trabajo asociado).

En lo que se refiere al empleo privado, la LGDPCD establece en el artículo 42 que las empresas que cuenten con una plantilla que supere las 50 personas trabajadoras deberán contratar al menos el $2 \%$ de personas con discapacidad. Se trata de una cuota de reserva de puestos de trabajo para el colectivo que este tipo de organizaciones deberán cumplir.

Según datos del $\mathrm{INE}^{9}$, en la CAE, unas 8.000 personas con discapacidad trabajan por cuenta ajena en el mercado privado, que equivale al $33 \%$ del total de las personas con discapacidad que se encuentran ocupadas.

En el ámbito del empleo ordinario público, debe acudirse al artículo 59 del $\mathrm{EBEP}^{10}$, el cual fija dos de los puntos más importantes en materia de empleo para personas con discapacidad. En primer lugar, establece que "En las ofertas de empleo público se reservará un cupo no inferior al $7 \%$ de las vacantes para ser cubiertas entre personas con discapacidad, (...) siempre que superen los procesos selectivos y acrediten su discapacidad y la compatibilidad con el desempeño de las tareas, de modo que progresivamente se alcance el $2 \%$ de los efectivos totales en cada Administración Pública". En segundo lugar, subraya que "Cada Administración pública adoptará las medidas precisas para establecer las adaptaciones y ajustes razonables de tiempos y medios en el proceso selectivo y, una vez superado dicho proceso, las adaptaciones en el puesto de trabajo a las necesidades de las personas con discapacidad".

En cuanto a los datos disponibles en el territorio ${ }^{11}$, se calcula que en el año 2016 casi 5.000 personas con discapacidad trabajaban en el empleo público, lo que supone un $20 \%$ del total de personas con discapacidad ocupada en la CAE.

En el caso del empleo autónomo, la realidad es complicada para el colectivo, y así lo demuestra la escasa demanda que tiene por parte de las personas con discapacidad. Ante esta situación, el artículo 47 de la LGDPCD requiere a los poderes públicos a que adopten "politicas de fomento del trabajo autónomo de personas con discapacidad dirigidas al establecimiento y desarrollo de iniciativas económicas y profesionales por cuenta propia, o a través de entidades de la economía social".

9. Instituto Nacional de Estadística.

10. Real Decreto Legislativo 5/2015, de 30 octubre, por el que se aprueba el texto refundido de la Ley del Estatuto Básico del Empleado Público.

11. Datos facilitados por el Observatorio Vasco de Economía Social. 
Los datos son el claro reflejo de la realidad, y muestran que el empleo autónomo de las personas con discapacidad en la CAE se contaría en unas 2.600 personas aproximadamente ${ }^{12}$.

Por último, se debe destacar el autoempleo colectivo o trabajo asociado, que se lleva a cabo en cooperativas o sociedades laborales. Al igual que el empleo autónomo, es un modelo poco utilizado, aunque en los últimos años, cada vez son más las personas que han optado por esta fórmula ${ }^{13}$. Se trata de una figura que fomenta la participación de las personas con discapacidad en la entidad de la que son propietarias.

\subsection{El empleo en Centros Especiales de Empleo}

Llegados a este punto, se centrará la atención en las características que definen a los Centros Especiales de Empleo dentro de la modalidad del empleo protegido, para, más adelante, poder analizar con más detalle el Modelo Vasco de Inclusión Sociolaboral, gestionado por CEE de iniciativa social.

Los Centros Especiales de Empleo son uno de los principales instrumentos facilitadores del acceso al empleo de las personas con discapacidad, y, por consiguiente, de la inclusión social del colectivo.

El artículo 43.1 de la LGDPCD se encarga de definir jurídicamente este tipo de entidades de la siguiente manera: "Los centros especiales de empleo son aquellos cuyo objetivo principal es el de realizar una actividad productiva de bienes o de servicios, participando regularmente en las operaciones del mercado, y tienen como finalidad el asegurar un empleo remunerado para las personas con discapacidad; a la vez que son un medio de inclusión del mayor número de estas personas en el régimen de empleo ordinario. Igualmente, los centros especiales de empleo deberán prestar, a través de las unidades de apoyo, los servicios de ajuste personal y social que requieran las personas trabajadoras con discapacidad, según sus circunstancias y conforme a lo que se determine reglamentariamente". Mediante este precepto, el legislador aclara que la finalidad es asegurar un empleo productivo y remunerado a las personas con especiales necesidades de apoyo, al mismo tiempo que facilitan el tránsito al mercado de empleo ordinario. Los CEE, desde su creación, trabajan por hacer real la inserción de las personas con discapacidad en el mercado de trabajo ordinario, siendo el objetivo principal facilitar el paso desde los $\mathrm{CEE}$ al EO ${ }^{14}$.

12. Datos facilitados por el Observatorio Vasco de Economía Social.

13. No se dispone de datos oficiales sobre el autoempleo colectivo de las personas con discapacidad.

14. La Ley anterior, LISMI 13/1982, ya establecía en el artículo 37 que la finalidad primordial de la política de empleo de trabajadores con discapacidad es su integración en el sistema de trabajo ordinario. 
Para analizar los aspectos fundamentales de este tipo de entidades, debe acudirse al Real Decreto 2273/1985, de 4 de diciembre, por el que se aprueba el Reglamento de los Centros Especiales de Empleo. El artículo 2 menciona que "sin perjuicio de la función social que los Centros Especiales de Empleo han de cumplir y de sus peculiares características, su estructura y organización se ajustará a los de las Empresas ordinarias".

Los CEE deberán cumplir, según el artículo 43 de la LGDPCD, dos requisitos obligatorios. Por un lado, la plantilla estará constituida, como mínimo, por el 70\% de personas trabajadoras con discapacidad, dentro del cual entrarían las personas con un grado de discapacidad reconocido igual o superior al 33\%. Y, por otro lado, todos los CEE deberán prestar, a través de las unidades de apoyo, los servicios de ajuste personal y social que requieran cada una de las personas trabajadoras y sus circunstancias ${ }^{15}$.

Cabe destacar que los CEE pueden clasificarse de tres maneras diferentes. En primer lugar, teniendo en cuenta si son de titularidad privada o pública ${ }^{16}$; en segundo lugar, en si tienen o no ánimo de lucro ${ }^{17}$; y, por último, en si son de iniciativa social o empresarial. Es esta última clasificación, ańadida recientemente, la que debemos resaltar en el presente texto. Se basa en la reciente modificación del artículo 43 de la LGDPCD, operada por la Disposición Final decimocuarta de la Ley 9/2017, de 8 de noviembre, por la cual se añadió el punto 4 que define por primera vez los CEE de iniciativa social (CEEis). Dicho precepto establece que "Tendrán la consideración de Centros Especiales de Empleo de iniciativa social aquellos que cumpliendo los requisitos que se establecen en los apartados $1 .^{\circ}$ y $2 .^{\circ}$ de este artículo son promovidos y participados en más de un 50 por ciento, directa o indirectamente, por una o varias entidades, ya sean públicas o privadas, que no tengan ánimo de lucro o que tengan reconocido su carácter social en sus Estatutos, (...) y se obliguen a la reinversión integra de sus beneficios para creación de oportunidades de empleo para personas con discapacidad y la mejora continua de su competitividad y de su actividad de economía social, teniendo en todo caso la facultad de optar por reinvertirlos en el propio centro especial de empleo o en otros centros especiales de empleo de iniciativa social". El precepto, además de definir jurídicamente

15. Según el artículo 43, "se entenderán por servicios de ajuste personal y social los que permitan ayudar a superar las barreras, obstáculos o dificultades que las personas trabajadoras con discapacidad de los centros especiales de empleo tengan en el proceso de incorporación a un puesto de trabajo, asi como en la permanencia y progresión en el mismo. Igualmente se encontrarán comprendidos aquellos dirigidos a la inclusión social, cultural y deportiva".

16. El artículo 45 de la LGDPCD establece que los CEE pueden ser creados por organismos públicos o privados.

17. El artículo 5.b del Real Decreto 2273/1985 establece que se atenderá a la aplicación de sus posibles beneficios. Si la aplicación es al objeto social o a la propia entidad, será considerada sin ánimo de lucro; por el contrario, si van dirigidos a otra finalidad, se considerará con ánimo de lucro. 
este tipo de entidades, fija las particularidades que deben cumplir. La modificación supuso un gran paso para los Centros Especiales de Empleo de iniciativa social, y, por ende, para los pertenecientes al Modelo Vasco de Inclusión Sociolaboral, tal y como se comprobará en el siguiente punto del estudio.

En cuanto a las cifras referentes a los $\mathrm{CEE}$, deben subrayarse los siguientes.

Tabla 1.

Empleo de las personas con discapacidad en CEE, CAE, 2019

\begin{tabular}{|l|c|c|c|}
\hline Año & CEEis & Resto CEE & Total en CEEis \\
\hline 2019 & $82 \%$ & $18 \%$ & 8.229 \\
\hline
\end{tabular}

Fuente: Elaborado a partir de Lanbide y EHLABE.

Tal y como muestran estos datos, en el año 2019 en la CAE 8.229 personas con discapacidad se encontraban empleadas en CEEis, esto supone el $82 \%$ de las personas con discapacidad empleadas en un CEE.

Es indudable el aporte que estas entidades realizan para generar oportunidades a las personas con discapacidad, desempeñan un papel fundamental en el desarrollo profesional del colectivo, y, en consecuencia, en su inclusión social. A pesar de esto, la realidad muestra las grandes dificultades a las que se enfrentan diariamente estas personas en el ámbito del empleo, y así lo reflejan los datos.

Según los datos del 2018 recogidos en el Instituto Nacional de Estadística ${ }^{18}$, la tasa de actividad de las personas con discapacidad en la CAE es del 34,9\%, mientras que el de la población sin discapacidad es del $56,27 \%$. En lo que se refiere a la tasa de paro, que comprende a las personas que buscan activamente empleo, también los indicadores de las personas con discapacidad son peores que el de las personas sin discapacidad. Mientras que en el primero de los casos se encuentra en el 16,5\%, en el segundo está en el 9,6\%. Estos datos son muy preocupantes y muestran la evidente necesidad de fomentar medidas de acción positiva desde diferentes sectores de la sociedad.

Al hilo de esto, resulta interesante mostrar los datos del indicador AROPE ${ }^{19}$ para el estado español (no están disponibles a nivel de la CAE), que mide el riesgo de pobreza y/o exclusión social. Las cifras muestran que en el 2018 tres de cada diez per-

18. Recogidos también en el Informe ODISMET 5 del Observatorio sobre discapacidad y Mercado de trabajo en España.

19. Indicador "At Risk Of Poverty and/or Exclusion" introducido por la Unión Europea. Considera el riesgo de pobreza, la baja intensidad laboral y la privación en el acceso a ciertos bienes y servicios básicos. 
sonas con discapacidad estaba en situación de pobreza o exclusión social, igual que al inicio de la crisis en el 2008. Mientras que la tasa fluctúa en el caso de la población total según el ciclo económico, no ocurre lo mismo en el caso de las personas con discapacidad y se mantiene en los mismos valores.

Es evidente que todavía hay mucho camino que recorrer para equiparar mínimamente la realidad social y laboral de las personas con discapacidad, más teniendo en cuenta los datos analizados. Por esta razón, es tan importante que los CEE sigan trabajando por mejorar la situación de las personas con discapacidad, para que pueden ejercer sus derechos con libertad y en igualdad de condiciones que las demás.

\subsection{Servicio ocupacional}

Por último, la LGDPCD prevé en el artículo 52 la opción del servicio ocupacional, cuyo objetivo principal es el desarrollo personal de las personas que participan en el programa, y en la medida que sea posible, trabajar en su capacitación y preparación para el acceso al empleo. Se trata de un servicio social, dirigido a las personas que a causa de la severidad de su discapacidad, no están capacitadas para el empleo.

La LGDPCD establece que también podrá dirigirse a personas trabajadoras con discapacidad que habiendo desarrollado una actividad laboral específica no hayan conseguido una adaptación satisfactoria o hayan sufrido un empeoramiento que haga aconsejable su integración en un centro ocupacional.

En la CAE, cohabitan el servicio ocupacional y el empleo en Centros Especiales de Empleo, se trata de un sistema que ha demostrado tiene grandes beneficios para las personas, ya que ofrecen un entorno ya conocido que ofrece seguridad y mejora el tránsito al empleo tanto en CEE como ordinario. Cabe resaltar, que la gran mayoría de las personas con discapacidad intelectual que hoy tienen un empleo en CEE provienen del servicio ocupacional ${ }^{20}$.

Tal y como se puede observar, la evolución ha sido positiva, ya que en los últimos años ha ido aumentando el número de personas que se encontraba en un servicio ocupacional, llegando a ser en el 2018 casi 2.400 personas. Por el contrario, estas cifras demuestran la brecha que existe también en este ámbito entre hombres y mujeres, ya que el $66 \%$ de las plazas son ocupadas por hombres. 


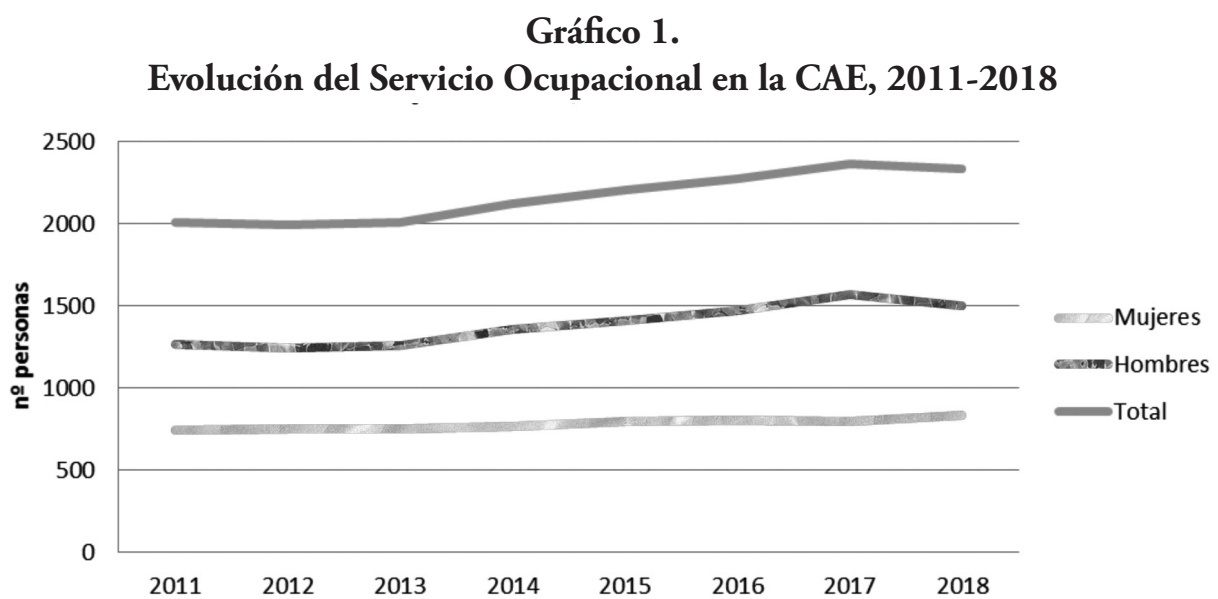

Fuente: Elaborado por el Observatorio Vasco de Economía Social (OVES-GEEB), a partir de los datos facilitados por EHLABE.

\section{Los Centros Especiales de Empleo de iniciativa social en la Comunidad Autónoma de Euskadi. El Modelo Vasco de Inclusión Sociolaboral}

Como se puede observar en los datos analizados (Tabla 1), una de las principales características del empleo en CEE en la CAE consiste en la gran importancia cuantitativa que los CEEis tienen en el ámbito laboral de las personas con discapacidad, ya que casi el $82 \%$ está empleada en CEEis, frente al $18 \%$ que se encuentra en un CEE con ánimo de lucro. Es por esta razón por la que resulta interesante analizar el Modelo Vasco de Inclusión Sociolaboral, gestionado por CEEis, y en el que se concentran el 95\% de todas las iniciativas de empleo de Euskadi.

Todas las entidades pertenecientes al modelo están agrupadas en torno a $\mathrm{EHLABE}^{21}$, la asociación vasca de entidades no lucrativas, que fomentan la inclusión sociolaboral de las personas con discapacidad. Actualmente, cuentan con más de 10.000 personas con discapacidad empleadas, ocupadas o participantes en los servicios de empleabilidad ${ }^{22}$.

21. EHLABE (Euskal Herriko Lan Babestuaren Elkartea). Concentra el 95\% de todas las iniciativas de empleo de la CAE, formado por 14 entidades y más de 100 centros de trabajo.

22. Datos facilitados por EHLABE. 
Los CEE pertenecientes al modelo vasco tienen un objetivo común, que es la generación de empleo de calidad ${ }^{23}$ para personas con discapacidad, especialmente para aquellas con más necesidades de apoyo. Entienden el trabajo como medio para la inclusión social del colectivo, convirtiéndolo en una herramienta muy importante en los procesos de plena inclusión. Además, todas comparten cada uno de los principios y valores de la Economía Social, con el objetivo de lograr una economía más sostenible y una sociedad más cohesionada.

El Modelo Vasco de Inclusión Sociolaboral se ha convertido en un referente en el empleo de las personas con discapacidad ${ }^{24}$. Una de las principales razones es que se centra en las capacidades de cada una de las personas, y diseña itinerarios profesionales con un enfoque inclusivo, priorizando a las personas con mayores necesidades de apoyo. Cabe destacar que uno de los principales objetivos de los CEEis pertenecientes a EHLABE es lograr la inserción del colectivo en el mercado laboral ordinario a través del empleo con apoyo, y es por esta razón por la que gestionan itinerarios profesionales adaptados a las necesidades de cada persona.

A continuación, se analizarán concisamente las principales características del Modelo Vasco de Inclusión Sociolaboral (Moratalla, P., 2017: 136):

- Los CEEis gestionan programas enfocados a la empleabilidad de las personas. Para ello, diseñan itinerarios completos de inserción laboral, dentro de los cuales se encuentran la orientación profesional, formación, intermediación laboral, etc. Además, cuentan con la colaboración de diferentes Administraciones públicas, empresas y asociaciones.

- Los itinerarios se diseñan con un enfoque inclusivo y adaptados a las necesidades y deseos de cada una de las personas. Se trata de un modelo flexible e innovador, que busca potenciar las capacidades de cada una de ellas.

- Todas las entidades pertenecientes al modelo vasco trabajan a favor de un empleo inclusivo, abierto, accesible y en igualdad de condiciones. Por esta razón, los servicios de empleabilidad se centran en cada una de las personas con discapacidad,

23. El empleo de calidad encaja con el concepto de trabajo decente o digno de la Organización Internacional del Trabajo, que establece que "el trabajo decente sintetiza las aspiraciones de las personas durante su vida laboral. Significa la oportunidad de acceder a un empleo productivo que genere un ingreso justo, la seguridad en el lugar de trabajo y la protección social para las familias, mejores perspectivas de desarrollo personal e integración social, libertad para que los individuos expresen sus opiniones, se organicen y participen en las decisiones que afectan sus vidas, y la igualdad de oportunidades y trato para todos, tanto para hombres como mujeres".

24. Los datos analizados sobre el empleo en CEEis así lo demuestran. Contribuyen de manera directa en la inclusión del colectivo. Esto se plasma en las acciones y servicios concretos que realizan, y en la mayor atención y esfuerzo que dedican en la mejora de la situación de las personas con mayores necesidades de apoyo. 
con el objetivo de que sean ellas las que elijan con libertad el entorno en el que desean trabajar.

- Trabajan por la excelencia en la gestión de diferentes tipos de actividades empresariales en entornos altamente competitivos y profesionales. Han generado empleo para personas con discapacidad en diferentes sectores de actividad, demostrando la capacidad de las personas con discapacidad para desempeñar diferentes trabajos.

- Por último, cabe resaltar el trabajo en red y la coordinación entre los CEEis y las Administraciones públicas y los agentes públicos, económicos y sociales.

El trabajo que llevan a cabo los CEEis en la CAE es fundamental para el colectivo de las personas con discapacidad, y por consiguiente, para la inclusión real de todas ellas en la sociedad. Muestra de ello, son algunos de los datos disponibles (Tabla 2).

Tal y como muestran los datos en la Tabla 2, el porcentaje de personas con discapacidad empleadas en los CEEis de Euskadi ha estado en los últimos años entre el $83 \%$ y el $90 \%$. Además, cabe destacar el aumento que han experimentado las tasas de empleo de las personas con más necesidades de apoyo, que son las personas con discapacidad psíquica.

También resulta interesante el dato sobre el tránsito del empleo desde los CEEis al empleo ordinario ${ }^{25}$, uno de los principales objetivos de estas entidades. En la CAE entre los años 2015 y 2018, la evolución ha sido muy positiva, mientras que en el 2015 fueron 49 las personas que transitaron de los CEEis al mercado ordinario, en el 2018 fueron 217 personas. Si bien es cierto que el número de personas que han transitado no es alto y el alcance es limitado, es indudable el trabajo que realizan las propias entidades para que ese número vaya en aumento y para generar oportunidades para que las personas puedan elegir libremente el entorno en el que desean desempeñar su trabajo.

\subsection{El modelo vasco a la luz de la Convención de la ONU}

Los CEEis de Euskadi no han sido ajenos a los cambios surgidos en los últimos ańos en materia de empleo y discapacidad. Tanto es así que la aprobación de la Convención de las Naciones Unidas sobre los derechos de las personas con discapacidad supuso un antes y un después en el modelo de tratamiento de la discapacidad y de los derechos del colectivo. 


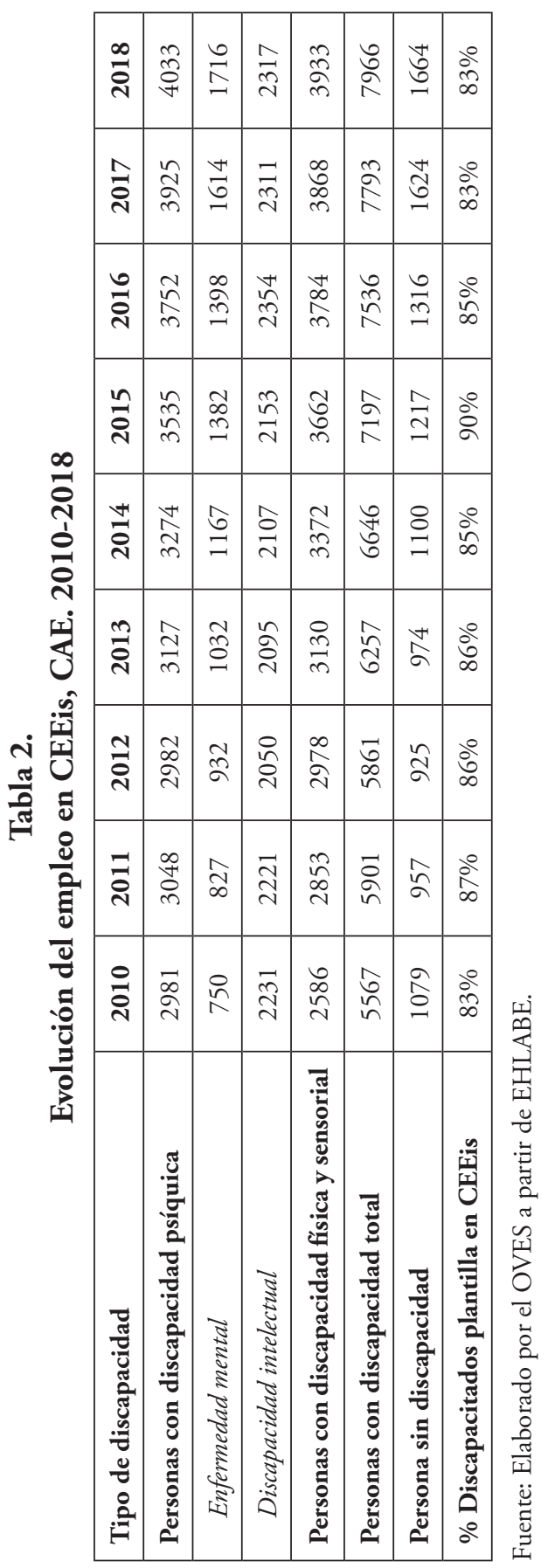


La Convención también marcó un punto de inflexión en materia de empleo, ya que se trata de un modelo en el que las dificultades de las personas con discapacidad para acceder al empleo son el resultado de la interacción entre las características de estas y las barreras que existen en el empleo en particular. El artículo 27 establece el derecho de las personas con discapacidad a trabajar y a ganarse la vida en igualdad de condiciones con las demás, mediante un trabajo libremente elegido en un entorno abierto, inclusivo y accesible.

Resulta interesante analizar el Modelo Vasco de Inclusión Sociolaboral a la luz del artículo 27 de la Convención Internacional, de manera que se puede identificar la relación entre los dos modelos.

La Convención subraya que el entorno laboral debe ser abierto, inclusivo y no discriminatorio. Esto significa que ninguna persona pueda ser excluida por las razones que sean, por lo que se deberán eliminar la discriminación y las barreras y adaptar los entornos a las personas. Por otro lado, para que el mercado laboral sea inclusivo, resulta necesario que la sociedad también lo sea, y, para ello, es fundamental que las propias personas con discapacidad participen en el proceso de elaboración de medidas relacionadas con sus derechos. Por último, es importante que los Estados y las Administraciones públicas promuevan medidas de acción positiva y prohíban todo tipo de discriminación por motivos de discapacidad relativas a cualquier forma de empleo.

Los CEEis de la CAE comparten con el la Convención el objetivo de promover un entorno laboral abierto, accesible y no discriminatorio. Para conseguir estos objetivos, gestionan programas enfocados a la mejora de la empleabilidad, con el fin de que sean las propias personas las que puedan elegir el trabajo mediante el que desean ganarse la vida. Se trata de una herramienta innovadora, que busca la potenciación de las capacidades de las personas con discapacidad y facilita el paso al mercado de trabajo ordinario. Por lo tanto, es un modelo centrado en las personas y en su desarrollo personal y profesional.

Además, como ya se ha mencionado anteriormente, las entidades trabajan gestionando actividades empresariales en entornos altamente competitivos y profesionales, generando puestos de trabajo para el colectivo en casi todos los sectores, demostrando las capacidades de las personas con discapacidad para desempeńar diferentes trabajos. De esta manera, adquieren experiencia laboral para poder transitar al empleo ordinario.

Otra de las particularidades del modelo vasco es el trabajo coordinado y en red con diferentes agentes públicos, sociales y económicos, como las Administraciones 
públicas $^{26}$, el Tercer Sector de Euskadi ${ }^{27}$, las Universidades, los Servicios Sociales, las entidades de la discapacidad, etc. Con el apoyo de todos estos agentes, se gestionan, apoyan y conforman los diferentes itinerarios sociolaborales.

Los CEEis agrupados en EHLABE, con más de 30 años de experiencia en el sector, han conseguido crear un entorno laboral abierto, accesible y no discriminatorio para las personas con discapacidad, en el cual pueden desarrollar sus capacidades en igualdad de condiciones y en total libertad, un ejemplo del modelo que defiende la Convención Internacional sobre los derechos de las personas con discapacidad.

\section{Conclusiones}

El empleo es uno de los instrumentos más importantes para la inclusión social de las personas con discapacidad, un derecho universal que proporciona autonomía, independencia e integración.

En el caso de Euskadi, tal y como muestran los datos, es relevante el papel que juegan los CEEis en el empleo del colectivo, no sólo por las oportunidades de trabajo que brindan, sino por haberse convertido en un instrumento para la visibilización del colectivo. El trabajo llevado a cabo por las entidades agrupadas en EHLABE ha convertido el Modelo Vasco de Inclusión Sociolaboral en un referente y ejemplo de buenas prácticas.

Durante el análisis, se ha podido comprobar cómo los CEEis contribuyen de manera directa a la inclusión de las personas con discapacidad, en especial, de las personas con más necesidades de apoyo, y trabajan a favor de un empleo digno y de calidad. A pesar de esto, siguen siendo varios los retos a los que se enfrentan de cara al futuro, con el fin de lograr más y mejor empleo para el colectivo.

Uno de los logros más importantes del modelo vasco es el entorno laboral que han construido, el cual está directamente relacionado con el modelo que defiende la Convención de las Naciones Unidas, el que las personas con discapacidad puedan elegir libremente el trabajo que desean desarrollar.

Deben subrayarse varios factores que han resultado clave para el buen posicionamiento del modelo. Por un lado, los CEEis entienden la realización de una actividad laboral como la herramienta más efectiva para la inclusión del colectivo. Además, fomentan la participación de las personas en la gestión y organización de cada una

26. Gobierno Vasco, Diputaciones Forales, Servicio Vasco de Empleo, INEM.

27. Organizaciones de iniciativa ciudadana y sin ánimo de lucro, que impulsan el interés general y la defensa de los derechos de todas las personas, con especial atención a aquellas que están en situación de mayor vulnerabilidad. 
de las entidades. Por otro lado, han trabajado siempre en entornos competitivos y con una gestión altamente profesionalizada, y sin olvidar la presencia que tienen en la mayoría de los sectores de actividad. Por último, resulta importante destacar el trabajo coordinado con los diferentes agentes públicos, sociales y económicos, que ha resultado ser fundamental para conseguir buenos resultados.

Sin embargo, los datos también han demostrado que la situación laboral del colectivo sigue sin ser igual al del resto de la población. Es por esta razón por la que es necesario seguir trabajando para seguir generando empleo de mayor calidad, que sea más accesible y abierto, como uno de los principales ejes de su inclusión en la sociedad.

Por lo tanto, los CEEis de Euskadi tienen varios retos por delante. En primer lugar, seguir trabajando en la provisión de apoyos para mejorar la transición al mercado ordinario de trabajo y el acceso al empleo (formación, orientación profesional, etc). Además, resulta fundamental trabajar para crear prácticas innovadoras que generen nuevas oportunidades laborales y adaptadas a los nuevos cambios que vienen con los avances de la tecnología.

En cuanto a la formación, es muy importante reducir la brecha que existe entre la educación obligatoria y la formación profesional y mejorar el acceso a la Universidad. Para ello, se deberán desarrollar apoyos y adaptaciones dentro de los espacios normalizados. Se trata de un punto clave para mejorar el acceso al empleo en general de las personas con discapacidad.

En lo relativo a los procesos de envejecimiento y/o deterioro prematuro de las personas con discapacidad, es necesario hacer esfuerzos para favorecer el envejecimiento activo y la calidad de vida en el empleo. También es muy importante la doble discriminación que sufren las mujeres con discapacidad; por esta razón, deben realizarse esfuerzos para hacer frente a esta situación mediante acciones de visibilización, sensibilización y empoderamiento, que ayuden a disminuir la situación de desigualdad en la que viven.

Por último, es necesario fomentar dentro de las Administraciones públicas e Instituciones figuras de representación del colectivo, de manera que participen de forma activa en todas las decisiones y medidas que se adopten entorno a ellas. 
(pp. 153-175)

\section{Bibliografía}

BENGOETXEA, A. (2014): "Economía Social e inserción laboral de las personas con discapacidad", Revista Vasca de Administración Pública, 99-100, 529-547.

BENGOETXEA, A., ECHEBARRIA, A., MORANDEIRA, J. \& SOTO, A. (2019): El Modelo Vasco de Inclusión Sociolaboral, UPV/EHU, Donostia

DE FUENTES, C. (2016): "Sobre el concepto jurídico de persona con discapacidad y la noción de apoyos necesarios", Revista Española de Discapacidad, 4 (2): 81-99

MORATALLA, P. (2017): "El modelo vasco de inclusión sociolaboral de personas con discapacidad”, Revista Vasca de Economía Social, 14, 129-144.

MORATALlA, P. (2016): "Centros Especiales de Empleo", CIRIEC-España, Revista Jurídica de Economia Social y Cooperativa, 29, 235-272.

TOBOSO, M. \& ARNAU, M.S. (2008): "La discapacidad dentro del enfoque de capacidades y funcionamientos de Amartya Sen”, Revista Iberoamericana Filosofía, Politica y Humanidades, (10(20)).

OBSERVATORIO VASCO DE ECONOMÍA SOCIAL (2018): "Informe anual de la situación de la economía social vasca”, OVES/GEEB.

\section{Legislación consultada}

Constitución Española (BOE, 29 de diciembre) https://www.boe.es/eli/es/c/1978/12/27/(1)

Directiva 2000/78/CE del Consejo, de 27 de noviembre de 2000, relativa al establecimiento de un marco general para la igualdad de trato en el empleo y la ocupación. (DOCE, de 2 de diciembre)

https://www.boe.es/buscar/doc.php?id=DOUE-L-2000-82357

Convención Internacional sobre derechos de las personas con discapacidad. http://www.un.org/disabilities/documents/convention/convoptprot-s.pdf

Ley $9 / 2017$, de 8 de noviembre, de Contratos del Sector Público, por la que se transponen al ordenamiento jurídico español las Directivas del Parlamento Europeo y del Consejo 2014/23/UE y 2014/24/UE, de 26 de febrero de 2014. (BOE, de 9 de noviembre) https://www.boe.es/eli/es/1/2017/11/08/9/con 
Ley 39/2006, de 14 de diciembre, de Promoción de la Autonomía Personal y Atención a las personas en situación de dependencia. (BOE, de 15 de diciembre) https://www.boe.es/eli/es/1/2006/12/14/39

Ley 13/1982, de 7 de abril, de integración social de minusválidos. (BOE, de 30 de abril) https://www.boe.es/eli/es/1/1982/04/07/13

Real Decreto Legislativo 1/2013, de 29 de noviembre, por el que se aprueba el Texto Refundido de la Ley General de derechos de las personas con discapacidad y de su inclusión social. (BOE, de 3 de diciembre) https://www.boe.es/eli/es/rdlg/2013/11/29/1

Real Decreto Legislativo 5/2015, de 30 de octubre, por el que se aprueba el texto refundido de la Ley del Estatuto Básico del Empleado Público. (BOE, de 31 de octubre). https:/www.boe.es/eli/es/rdlg/2015/10/30/5/con

Real Decreto 2273/1985, de 4 de diciembre, por el que se aprueba el Reglamento de los Centros Especiales de Empleo definidos en el artículo 42 de la Ley 13/1982, de 7 de abril, de Integración Social del Minusválido. (BOE, de 9 de diciembre) https://www.boe.es/eli/es/rd/1985/12/04/2273

Real Decreto 290/2004, de 20 de febrero, por el que se regulan los enclaves laborales como medida de fomento del empleo de las personas con discapacidad. https://www.boe.es/buscar/pdf/2004/BOE-A-2004-3277-consolidado.pdf

\section{Páginas web consultadas}

EHLABE (Asociación de Empleo Protegido de Euskadi): http://www.ehlabe.org/es/ INE (Instituto Nacional de Estadística): www.ine.es

OVES/GEEB (Observatorio Vasco de Economía Social): www.oves-geeb.eus

OIT (Organización Internacional del Trabajo):

https://www.ilo.org/global/lang--es/index.htm 